\title{
Double-well magnetic trap for Bose-Einstein condensates
}

\author{
N. R. Thomas, ${ }^{1}$ C. J. Foot ${ }^{2}$ and A. C. Wilson ${ }^{1}$ \\ ${ }^{1}$ Physics Department, University of Otago, P.O. Box 56, Dunedin, New Zealand \\ ${ }^{2}$ Clarendon Laboratory, Department of Physics, University of Oxford, \\ Parks Road, Oxford, OX1 3PU, United Kingdom.
}

(Dated: August 10, 2001)

\begin{abstract}
We present a magnetic trapping scheme for neutral atoms based on a hybrid of Ioffe-Pritchard and Time-averaged Orbiting Potential traps. The resulting double-well magnetic potential has readily controllable barrier height and well separation. This offers a new tool for studying the behavior of Bose condensates in double-well potentials, including atom interferometry and Josephson tunneling. We formulate a description for the potential of this magnetic trap and discuss practical issues such as loading with atoms, evaporative cooling and manipulating the potential.
\end{abstract}

PACS numbers: 03.75.Fi, 39.25.+k, 85.70.Ay

Bose-Einstein condensates (BECs) of dilute alkali gases have been the subject of a great deal of attention since they were first realized 11, 2, 3. 3. One area in which there has been considerable theoretical interest is the behavior of Bose condensates confined in double-well potentials. The process of splitting a condensate in two has been investigated by raising a potential barrier in the center of a harmonic trap [4, 5, 6, 6, 8]. This work has given insight into the dynamic behavior of condensates and modification of collective excitations. Also extensively studied is the area of Josephson junctions [9, 10, 11, 12, 13, 14, where the condensate mean field affects the tunneling between wells and macroscopic quantum self-trapping is possible. Other researchers have proposed experiments involving the relative phase of the two condensates. For example, uniting the relative phase of two initially independent Bose condensates [15, 16, investigating quantum and thermal fluctuations of the phase 17], and detection of weak forces 18.

To date the only experimental demonstration of a double-well potential for Bose condensates has been a hybrid configuration involving a harmonic magnetic potential divided into two regions by a repulsive optical field [19]. This scheme was used to observe interference fringes of two overlapping Bose condensates, in the first demonstration of long range coherence. More recently, the same configuration was used to measure the presence of vortices induced in one of the wells [20]. One feature of the apparatus is that there is high degree of stability between the magnetic and optical fields.

In this work we address the problem of producing a purely magnetic double-well potential suitable for studying BECs. Our scheme is an extension of the existing Ioffe-Pritchard (IP) and Time-averaged Orbiting Potential (TOP) traps used in many BEC experiments. We begin by briefly summarizing the IP trap and review how a double quadrupole-like potential can be formed. Bose condensates cannot be confined in this potential, because of Majorana spin-flip loss, but we show how the addition of a rotating bias field can resolve this problem. The focus of this work is the development of a theoretical description of the resulting double-well magnetic potential.
Practical issues such as loading, evaporative cooling and controlling the shape of the potential are considered in detail.

\section{IOFFE-PRITCHARD TRAPS}

Ioffe-Pritchard (IP) traps 21, 22 have been used extensively in the realization of BEC in alkali gases. The basic configuration for an IP trap is illustrated in Fig. 1. Four long "bars" with currents in alternate directions run parallel to the $z$ axis. These generate a quadrupole field in the $x-y$ plane with gradient $B^{\prime}$, which leads to radial confinement. Two "pinch" coils have currents in the same direction and are spaced to give a harmonic local minimum of the axial magnetic field with curvature $B^{\prime \prime}$. These coils provide axial confinement but also a large bias field $B_{p}$. Finally there are the two "nulling" coils (usually in the Helmholtz configuration) which provide a uniform axial field $B_{n}$. These allow the total bias field

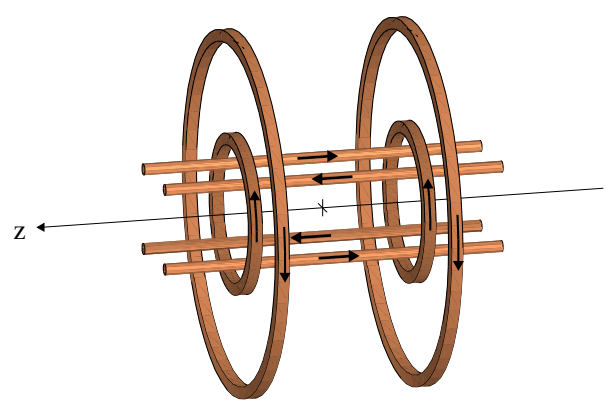

FIG. 1: The layout of coils and bars for a Ioffe-Pritchard magnetic trap with current direction indicated by arrows. The four bars lie on corners of a square. Current in each bar flows in the direction opposite to that of its closest neighbors, and the magnitude of the current is the same for each bar. The two pairs of coils with large and small radii are the nulling and pinch coils respectively. The currents flow in the opposite sense so that they produce opposing magnetic fields. 


\begin{tabular}{|c|c|c|c|c|c|}
\hline Ref. & Variant & Species & $\begin{array}{r}B_{o} \\
(\mathrm{G}) \\
\end{array}$ & $\begin{array}{r}B^{\prime} \\
(\mathrm{G} / \mathrm{cm}) \\
\end{array}$ & $\begin{array}{r}B^{\prime \prime} \\
\left(\mathrm{G} / \mathrm{cm}^{2}\right) \\
\end{array}$ \\
\hline 24 & Cloverleaf & ${ }^{23} \mathrm{Na}$ & 1 & 170 & 125 \\
\hline 25 & Baseball & ${ }^{87} \mathrm{Rb}$ & 1.6 & 300 & 85 \\
\hline 26 & Perm. Magnet & ${ }^{7} \mathrm{Li}$ & 1000 & 1250 & 855 \\
\hline 27 * & 4-Dee & ${ }^{23} \mathrm{Na}$ & 1.5 & 220 & 240 \\
\hline 28 & Traditional & ${ }^{87} \mathrm{Rb}$ & 1.5 & 275 & 365 \\
\hline 29 & QUIC & ${ }^{87} \mathrm{Rb}$ & 2 & 220 & 260 \\
\hline B0 & 3-coil & ${ }^{87} \mathrm{Rb}$ & 1.3 & 140 & 85 \\
\hline 31 & Superconductor & $\mathrm{H}$ & 0.5 & 240 & 0.75 \\
\hline 32 & Perm. Magnet & ${ }^{87} \mathrm{Rb}$ & 190 & 1200 & 400 \\
\hline $33 *$ & Baseball & ${ }^{87} \mathrm{Rb}$ & 1 & 175 & 62 \\
\hline 34 & Cloverleaf & ${ }^{87} \mathrm{Rb}$ & 1 & 175 & 185 \\
\hline 35 & QUIC & ${ }^{87} \mathrm{Rb}$ & 1.6 & 170 & 110 \\
\hline 36 & Cloverleaf & ${ }^{4} \mathrm{He}^{*}$ & 0.3 & 85 & 40 \\
\hline 37 & QUIC & ${ }^{4} \mathrm{He}^{*}$ & 4.2 & 280 & 200 \\
\hline
\end{tabular}

TABLE I: A summary of reported IP trap parameters. In many cases the traditional geometry of Fig. If is replaced by a variant with some specific advantages. References denoted * have field quantities calculated from the trap oscillation frequencies, and $\dagger$ indicates an equivalent radial gradient for traps that are harmonic.

at the trap center,

$$
B_{o}=B_{p}-B_{n}
$$

to be reduced. For BEC experiments this is important because it leads to tighter radial confinement and simplifies RF evaporation 23.

The magnetic field components for this geometry, to second order about the trap center, are given by the equations

$$
\begin{aligned}
& B_{x}=\quad+B^{\prime} x-\frac{1}{2} B^{\prime \prime} x z \\
& B_{y}=\quad-B^{\prime} y-\frac{1}{2} B^{\prime \prime} y z, \\
& B_{z}=B_{o} \\
& \quad+\frac{1}{2} B^{\prime \prime}\left(z^{2}-\frac{1}{2} r^{2}\right)
\end{aligned}
$$

where $r$ is the radial coordinate $\left(r^{2}=x^{2}+y^{2}\right)$. The terms with coefficient $B^{\prime}$ are generated by the four bars. Atoms in a weak-field seeking state are trapped at the minimum of the field magnitude. For small thermal atomic clouds and Bose-Einstein condensates $\left(k_{B} T<\mu B_{o}\right)$, the field magnitude is calculated by a binomial expansion to second order, to give

$$
B_{\mathrm{IP}}=B_{o}+\frac{1}{2}\left(\frac{B^{\prime 2}}{B_{o}}-\frac{B^{\prime \prime}}{2}\right) r^{2}+\frac{1}{2} B^{\prime \prime} z^{2},
$$

where $B_{o}>0$. The radial curvature is large when $B^{\prime 2} / B_{o} \gg B^{\prime \prime} / 2$, which is achieved by reducing $B_{o}$ with the nulling coils. A summary of reported parameters for various IP traps used in BEC experiments is shown in Table If. Typical values for the popular coil-based IP traps are $B_{o}=1 \mathrm{G}, B^{\prime}=200 \mathrm{G} / \mathrm{cm}$ and $B^{\prime \prime}=150 \mathrm{G} / \mathrm{cm}^{2}$. Note that the curvature is usually much larger radially (a)
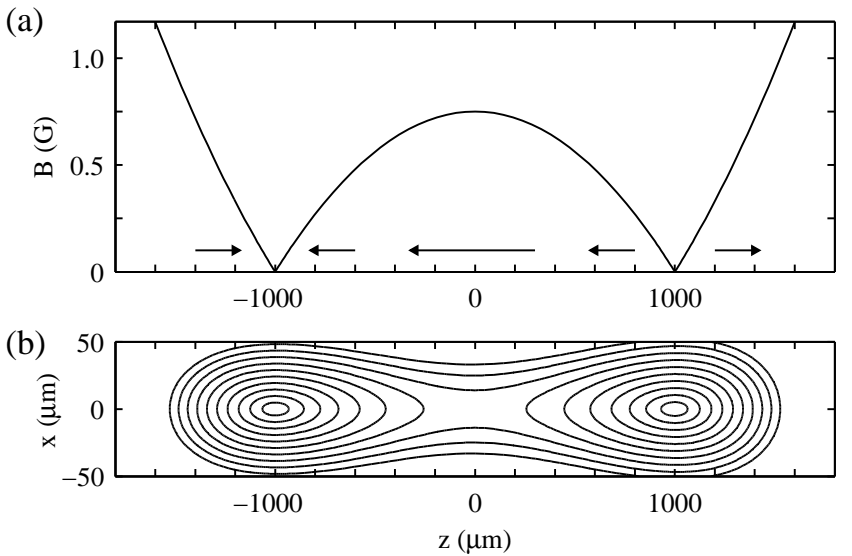

FIG. 2: A double-well potential with well spacing of $2 z_{o}=$ $2 \mathrm{~mm}$, generated by fields of $B_{o}=-0.75 \mathrm{G}, B^{\prime}=200 \mathrm{G} / \mathrm{cm}$ and $B^{\prime \prime}=150 \mathrm{G} / \mathrm{cm}^{2}$. (a) The behavior for the $z$ axis with the field directions indicated by arrows. (b) Field magnitude contours in the $x-z$ plane with $0.1 \mathrm{G}$ spacing. The barrier height is equivalent to a temperature $\left(=\mu_{B} \Delta B / k_{B}\right)$ of $50 \mu \mathrm{K}$ for atoms in a magnetic substate with $\mu=\mu_{B}$.

than axially so that atomic clouds are cigar shaped with their long axis in the $z$ direction. For the values above, the radial curvature is $B_{r}^{\prime \prime} \approx 40000 \mathrm{G} / \mathrm{cm}^{2}$ and the anisotropy is $\lambda \equiv \omega_{r} / \omega_{z}=\sqrt{B_{r}^{\prime \prime} / B_{z}^{\prime \prime}}=16.3$. In this calculation $\omega_{z}$ and $\omega_{r}$ are the axial and radial oscillation frequencies in the harmonic trap.

Ketterle et al. [38, 39] have pointed out that a doublewell is formed if the nulling bias $\left(B_{n}\right)$ is allowed to overcome the pinch bias $\left(B_{p}\right)$, so that $B_{o}<0$ in Eq. (11). The axial field now has zeros at the points $z_{ \pm}= \pm z_{o}$, where

$$
z_{o}=\sqrt{\frac{2\left|B_{o}\right|}{B^{\prime \prime}}}
$$

The trapping potential then has the form of two wells, as shown in Fig. 2. The barrier height $\Delta B$ is $\left|B_{o}\right|$, which occurs at $(z, r)=(0,0)$.

The field components, from Eq. (2) evaluated about the well bottom $z_{ \pm}$, are given by

$$
\begin{aligned}
B_{x} & =\left(+B^{\prime}-\frac{1}{2} B^{\prime \prime} z_{ \pm}\right) x \\
B_{y} & =\left(-B^{\prime}-\frac{1}{2} B^{\prime \prime} z_{ \pm}\right) y \\
B_{z} & = \\
B^{\prime \prime} z_{ \pm} & z^{\prime}+\frac{1}{2} B^{\prime \prime} z^{\prime 2},
\end{aligned}
$$

where $z^{\prime}=z-z_{ \pm}$. If we were to neglect the term in $z^{\prime 2}$, then these equations would be similar to those of a spherical quadrupole field. This approximation is reasonable near the bottom of each well, where $z^{\prime} / 2 z_{ \pm} \ll 1$. However, in general the curvature distorts the potential from a quadrupole. Note that the axial gradient scales with the well position $z_{o}$, so that confinement tightens and becomes more linear as the well separation increases. The gradients along the $x$ and $y$ axes are very similar because we will consider well separations small enough that $B^{\prime} \gg B^{\prime \prime} z_{o}$. The $x-y$ asymmetry is therefore small and 
can often be neglected, but we include it in subsequent calculations for completeness.

The usefulness of the potential in Fig. 2 for BECs is severely limited by Majorana spin flips at the two field zeros 40, 41. Removing this means of trap loss in the double-well trap above is the motivation behind this work.

\section{DOUBLE-TOP TRAP}

To eliminate loss from the two quadrupole-like wells, we apply the TOP trap scheme developed by Cornell et al. 40] for the simple quadrupole trap. We now consider adding a rotating bias field to the double-well IP trap. The rotating field component, with magnitude $B_{t}$, is chosen to be

$$
\begin{aligned}
& B_{x}=B_{t} \cos \omega t, \\
& B_{y}=B_{t} \sin \omega t, \\
& B_{z}=0
\end{aligned}
$$

The oscillating field has no $z$ component, which avoids the introduction of further radial asymmetry and modulation of the well spacing, stiffness, and barrier height during the bias rotation. This choice also leads to a relatively simple description of the trap. As in the standard TOP trap, the frequency of rotation $\omega$ must satisfy

$$
\omega_{z}, \omega_{r} \ll \omega \ll \omega_{L}
$$

where $\omega_{L}$ is the Lamor frequency in field $B_{t}$. The lower bound ensures a time-averaged potential, and the upper bound allows the atomic magnetic dipole to follow the oscillating field so that the atoms remain trapped. These limits cause no practical difficulties as trap oscillation and Lamor frequencies are normally of order $100 \mathrm{~Hz}$ and $1 \mathrm{MHz}$ respectively.

The key mechanism of the TOP trap is that the field zero of a spherical quadrupole is displaced outside the atomic cloud by the rotating bias field of Eq. (6). In the original implementation [40] the field rotated about the symmetry axis of a spherical quadrupole giving a circular locus of $B=0$ at radius $r_{o}=B_{t} / B^{\prime}$, where $B^{\prime}$ is the radial gradient of the quadrupole. The double-TOP trap described in this work does not in general have equal gradients in the plane in which the bias rotates, so the locus is slightly elliptical. The axial displacements for $x$ and $y$ are given by

$$
r_{i}=\frac{B_{t}}{\left|B_{i}^{\prime}\right|}
$$

where $i=\{x, y\}$ and $B_{i}^{\prime}=\frac{\partial B_{i}}{\partial i}$ is the magnetic field gradient on axis $i$ evaluated from Eq. (5). Some other implementations of the TOP trap 42, 43 also have an elliptical locus because the oscillating magnetic field does not rotate about the symmetry axis of a spherical quadrupole. The double-TOP will have a circular locus for the field (a)
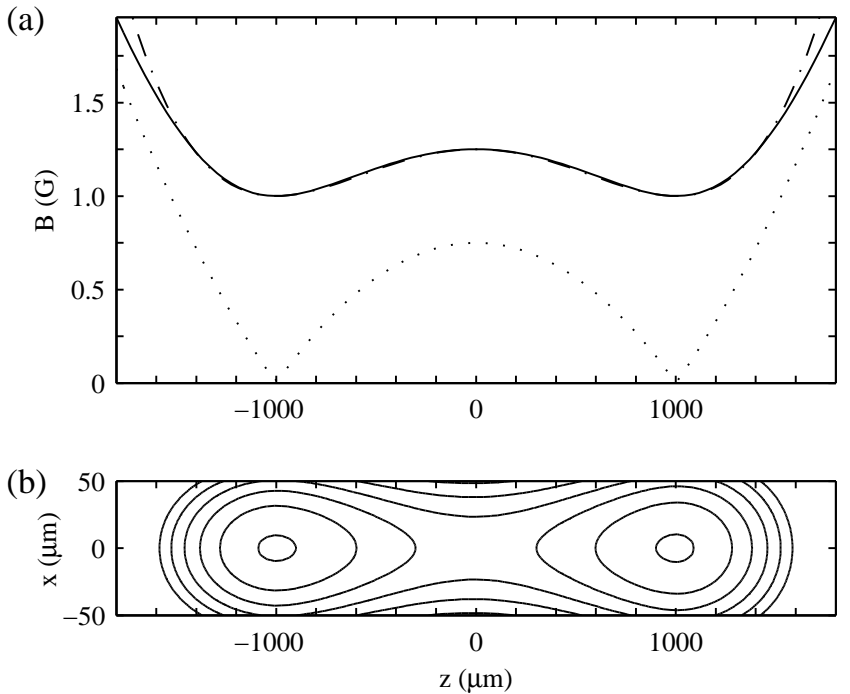

FIG. 3: A double-TOP potential with the same parameters as Fig. 2 $\left(2 z_{o}=2 \mathrm{~mm}, B_{o}=-0.75 \mathrm{G}\right)$ and $B_{t}=1 \mathrm{G}$. (a) The behavior on the $z$-axis with numerical (solid), Mexican-hat (dash-dot) and double-well IP (dotted) potentials shown. (b) A contour plot in the $x-z$ plane for the numerical integration with $0.1 \mathrm{G}$ spacing starting from the well bottom $B_{t}$. The barrier height is equivalent to $17 \mu \mathrm{K}$ for atoms with $\mu=\mu_{B}$.

zeros when the $x$-y asymmetry is negligible, corresponding to the limit $z_{o} \ll 1 \mathrm{~cm}$ for our typical parameters.

Following Cornell's method for the standard TOP trap, the field distribution for the double-TOP can be evaluated by averaging the field magnitude:

$$
B_{\mathrm{av}}=\frac{\omega}{2 \pi} \int_{0}^{2 \pi / \omega} B(t) d t
$$

where $B(t)$ is the instantaneous field magnitude. In Fig. 3 we have numerically evaluated Eq. 9. The addition of the rotating bias field to the double-well IP trap has displaced the field zero as required, leaving a minimum of field magnitude of $B_{t}$ occurring at $(z, r)=\left(z_{ \pm}, 0\right)$. The field at the top of the barrier separating the two wells is now the quadrature sum of static and rotating bias fields, given by

$$
B_{b}=\sqrt{B_{o}^{2}+B_{t}^{2}}
$$

The barrier height $\Delta B$ is then given by $\Delta B=B_{b}-B_{t}$. The well separation is not affected by the addition of the rotating bias field.

There is no straightforward analytical solution to Eq. (9) that applies for a wide range of parameters. However, it is possible to find results for restricted conditions and we now present two such cases. Along the central axis of the trap, the field magnitude can be approximated 
by a 1-dimensional Mexican-hat functional form

$$
B_{\mathrm{av}}(z)=B_{t}+\Delta B\left(1-\left(\frac{z}{z_{o}}\right)^{2}\right)^{2},
$$

and the error is less than $1.5 \%$ for $\left|B_{o}\right| / B_{t} \leq 1$ in the region $|z| / z_{o} \leq \sqrt{2}$. This description becomes more accurate as the ratio $\left|B_{o}\right| / B_{t}$ becomes smaller. Figure 3 shows that the fit is best in the central region where $B_{t} \leq B_{\text {av }}(z) \leq B_{b}$. At larger values of $z$ the timeaveraged field is dominated by the static axial component, so the potential becomes harmonic.

The second case is to consider the shape of each well near the minimum. This description applies for a cold atomic cloud confined within either well. We proceed by performing an analytical integration of Eq. (9), using the double-well fields expanded about $z= \pm z_{o}$ and the rotating bias. The resulting field magnitude is given by

$$
\begin{aligned}
B_{\mathrm{av}} \approx B_{t} & +\frac{1}{2}\left(\frac{B^{\prime 2}}{2 B_{t}}+\frac{\left|B_{o}\right| B^{\prime \prime}}{4 B_{t}}\right) r^{2}+\frac{B^{\prime \prime} B^{\prime}}{4 B_{t}} z_{ \pm}\left(y^{2}-x^{2}\right) \\
& +\frac{\left|B_{o}\right|}{B_{t}} B^{\prime \prime} z^{\prime 2}+\frac{B^{\prime \prime 2}}{2 B_{t}} z_{ \pm} z^{\prime 3}+\frac{B^{\prime \prime 2}}{8 B_{t}} z^{\prime 4}
\end{aligned}
$$

Examining this result we find that the radial dependence is harmonic and is dominated by the term $B^{\prime 2} /\left(4 B_{t}\right)$, which is identical to the standard TOP trap with field rotating in the $x-y$ plane. The term in $\left(y^{2}-x^{2}\right)$ is the result of the slight $x-y$ asymmetry of the double-well and is negligible for $z_{o} \ll 1 \mathrm{~cm}$. Compared to the single-well IP trap of Eq. (3) the axial curvature has been modified by the factor $2\left|B_{o}\right| / B_{t}$, so that axial confinement is tightened for $\left|B_{o}\right| \approx B_{t}$. The terms in $z^{\prime 3}$ and $z^{\prime 4}$ describe the barrier, and for our typical parameters these terms are important when $z_{o}$ is smaller than approximately $500 \mu \mathrm{m}$. If the wells are more widely spaced, the third order term adds only a slight tilt to the harmonic confinement and the 4 th order term can be neglected, as shown in Fig. 4.

In summary, the complete potential can be calculated numerically, while near the well minima can be described analytically. The Mexican-hat functional form is a useful approximation for the axial field magnitude in the central region.

\section{LOADING FROM A IOFFE-PRITCHARD TRAP}

The double-TOP trap may be loaded with a Bose condensate by transfer from a IP trap. To do this without spin-flip loss it is necessary to apply the rotating bias before reverse biasing to form a double-well. We model this loading scheme by adding the rotating bias of Eq. (6) to the IP field components of Eq. (2), and on integrating for the magnitude find that

$$
B_{\mathrm{av}}=B_{b}+\frac{1}{2}\left[\frac{B_{o}}{B_{b}}\left(\frac{B^{\prime 2}}{B_{o}}-\frac{B^{\prime \prime}}{2}\right)-\frac{B_{t}^{2} B^{\prime 2}}{2 B_{b}^{3}}\right] r^{2}
$$
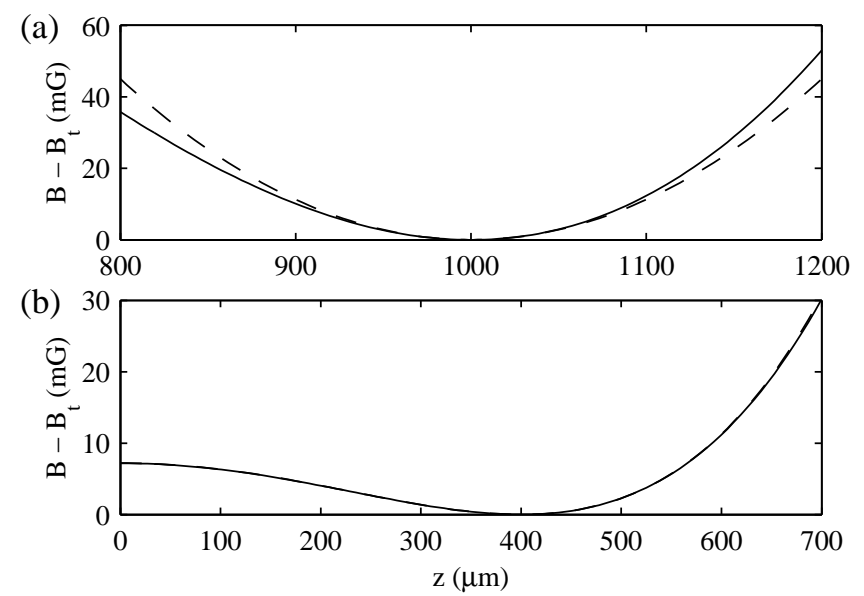

FIG. 4: Right-hand well of double-TOP potentials with common field parameters $B^{\prime}=200 \mathrm{G} / \mathrm{cm}, B^{\prime \prime}=150 \mathrm{G} / \mathrm{cm}^{2}$ and $B_{t}=1 \mathrm{G}$. Both the numerical average (solid) and evaluation of Eq. 12 (dashed) are shown. (a) The axial potential for widely separated wells $\left(z_{o}=1000 \mu \mathrm{m}\right)$ and relatively high barrier $(\Delta B=250 \mathrm{mG})$. Eq. 12 is evaluated to second order. (b) The axial potential for closely separated wells $\left(z_{o}=400 \mu \mathrm{m}\right)$ and a low barrier $(\Delta B=7.2 \mathrm{mG})$. The analytical result is taken to 4 th order in $z$.

$$
\begin{aligned}
& +B^{\prime} B^{\prime \prime} \frac{B_{o}^{2}+B_{b}^{2}}{4 B_{b}^{3}} z\left(y^{2}-x^{2}\right) \\
& +\frac{1}{2} \frac{B_{o}}{B_{b}} B^{\prime \prime} z^{2}+\frac{B_{t}^{2} B^{\prime \prime 2}}{8 B_{b}^{3}} z^{4} .
\end{aligned}
$$

Compared to the initial IP trap, both the axial and radial curvatures are reduced by at least a factor of $B_{o} / B_{b}$ due to the time-average. This effect can be minimized if $B_{b} \approx B_{o}$ which occurs when $B_{t}$ is small in comparison to $B_{o}$. Since $B_{o}>0$, there are no zeros of the field in this configuration and therefore there is no restriction on the minimum size of $B_{t}$. This choice of parameters also minimizes the effect of the third term in the radial curvature. A fourth order term in $z$ is required because the axial curvature goes to zero with $B_{o}$.

The loading process is an adiabatic evolution from IP to the time-averaged IP trap and finally to double-TOP. We can use Eq. (13) to describe most of this process, as shown in Fig. 5. The first step is to increase $B_{t}$ from 0 to $1 \mathrm{G}$ while holding $B_{o}=1 \mathrm{G}$. The trap minimum becomes $B_{b}=\sqrt{2} B_{o}$, and the axial and radial curvatures are reduced by factors of 0.71 and 0.53 respectively. A typical condensate is sufficiently small $(\approx 150 \mu \mathrm{m}$ long $)$ for the axial confinement to be harmonic. The second stage is to ramp $B_{o}$ down to $-0.75 \mathrm{G}$ which creates a double-TOP trap characterized by $\Delta B=250 \mathrm{mG}, 2 z_{o}=2000 \mu \mathrm{m}$. During this ramp the radial confinement is mostly unchanged because the rotating bias dominates $B_{b}$. When $B_{o}$ passes through zero, $B_{t}$ must be sufficiently large to place the field zero outside the cloud of atoms and prevent spin-flip loss. For the typical parameters used, the 

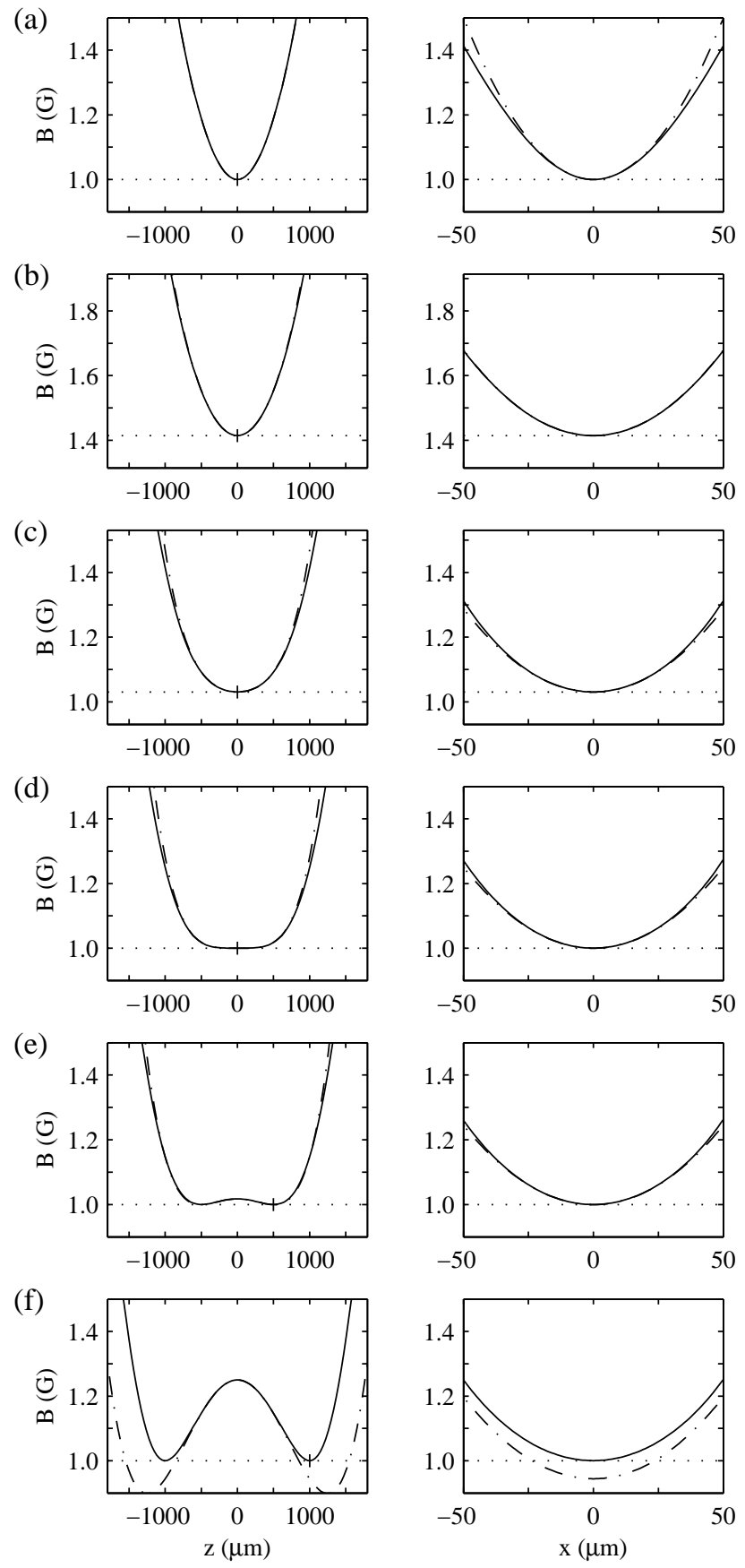

FIG. 5: Numerical (solid) and analytical (dash-dot) modeling of the transformation from IP to double-TOP trap by reducing $B_{o}$. The behavior is shown for the $z$-axis (left column), and $x$-direction at the trap minima (right column) indicated by the marker on the corresponding $z$-axis plot. The plots show: (a) The initial IP trap with $B_{o}=1 \mathrm{G}, B^{\prime}=200 \mathrm{G} / \mathrm{cm}$, $B^{\prime \prime}=150 \mathrm{G} / \mathrm{cm}^{2}, B_{t}=0 \mathrm{G}$; (b) The time-averaged IP formed with $B_{t}=1 \mathrm{G}$; (c)-(f) $B_{o}$ equal to $0.25 \mathrm{G}, 0 \mathrm{G},-0.19 \mathrm{G}$, and $-0.75 \mathrm{G}$ respectively (all other parameters held constant). The well separations in (e) and (f) are $2 z_{o}=1000 \mu \mathrm{m}$ and $2000 \mu \mathrm{m}$. The radii of the locus traced by the two quadrupole field zeros in (d)-(f) are constant and equal to $r_{x}=51.9 \mu \mathrm{m}$ and $r_{y}=48.2 \mu \mathrm{m}$. radial curvature of $20000 \mathrm{G} / \mathrm{cm}^{2}$ at $B_{o}=0$ means a typical alkali condensate of $10^{6}$ atoms will be significantly inside $r_{o}=50 \mu \mathrm{m}$. The ramp of $B_{o}$ produces a dramatic change in potential on the $z$ axis. While $B_{o}>0$, the curvature is positive but reducing, leading to a flattening as the quartic term becomes more important. Some control of the rate of change of the curvature is possible by the choice of $B_{t}$ relative to $B_{o}$. In this case, where they are approximately equal at start of the ramp, the curvature does not greatly deviate from a linear reduction, and this is also true for the case $B_{t}>B_{o}$. In the opposite extreme the curvature can collapse quite suddenly. When $B_{o}$ passes through zero, the sign of the curvature reverses and the barrier starts to rise. In Fig. $5(f)$ we see that although the analytical solution (Eq. (13)) describes the qualitative shape of the potential, there is no longer good quantitative agreement with the numerical result. The Mexican hat description is more appropriate for this situation.

A second method for loading Bose condensates into a double-TOP configuration is to evaporatively cool a thermal cloud. In this case the evaporation process is much the same as for a standard TOP trap, with the RF field oriented perpendicular to the rotating bias field, except that RF resonance occurs at two points at the perimeter of the cloud rather than one. Alternatively, an atomic cloud could be cooled first in a IP trap and then in a double-TOP. However in this case, the standard orientation for the RF antenna in a IP trap is not well matched for optimum evaporation in the double-TOP.

\section{CONTROLLING THE BARRIER HEIGHT AND WELL SPACING}

Careful control of the barrier height will be important in any investigation of condensate splitting and Josephson tunneling. Such studies are likely to require a barrier height comparable with the condensate chemical potential and therefore much smaller than that which we considered in the sections above. Recall that the barrier height is given by $\Delta B=\sqrt{B_{o}^{2}+B_{t}^{2}}-B_{t}$, so for the trap parameters associated with Fig. $5(\mathrm{f})$ we have $\Delta B=0.25 \mathrm{G}$. For atoms trapped in states with a magnetic dipole moment of $\mu_{B}$, this barrier height is equivalent to a temperature of $\mu_{B} \Delta B / k_{B}=16.8 \mu \mathrm{K}$. In comparison, the chemical potential, $\mu$ [44], of $10^{6}$ atoms of ${ }^{87} \mathrm{Rb}$ in the $\left|F=2, m_{F}=2\right\rangle$ state and confined in one of the (almost harmonic) wells is $\mu / k_{B}=180 \mathrm{nK}$, so that the ratio is $\mu_{B} \Delta B / \mu=93$.

The options for lowering the barrier height are to lower $B_{o}$ or increase $B_{t}$. Increasing $B_{t}$ keeps the well positions fixed but relaxes the radial confinement, while decreasing $\left|B_{o}\right|$ reduces the well spacing. In Fig. 6(a) we show the change in barrier height as a function of $B_{o}$ while keeping $B_{t}$ fixed. Initially the barrier is some 100 times higher than the chemical potential but decreases to a comparable height at $B_{o}=-55 \mathrm{mG}$. The chemical potential is 

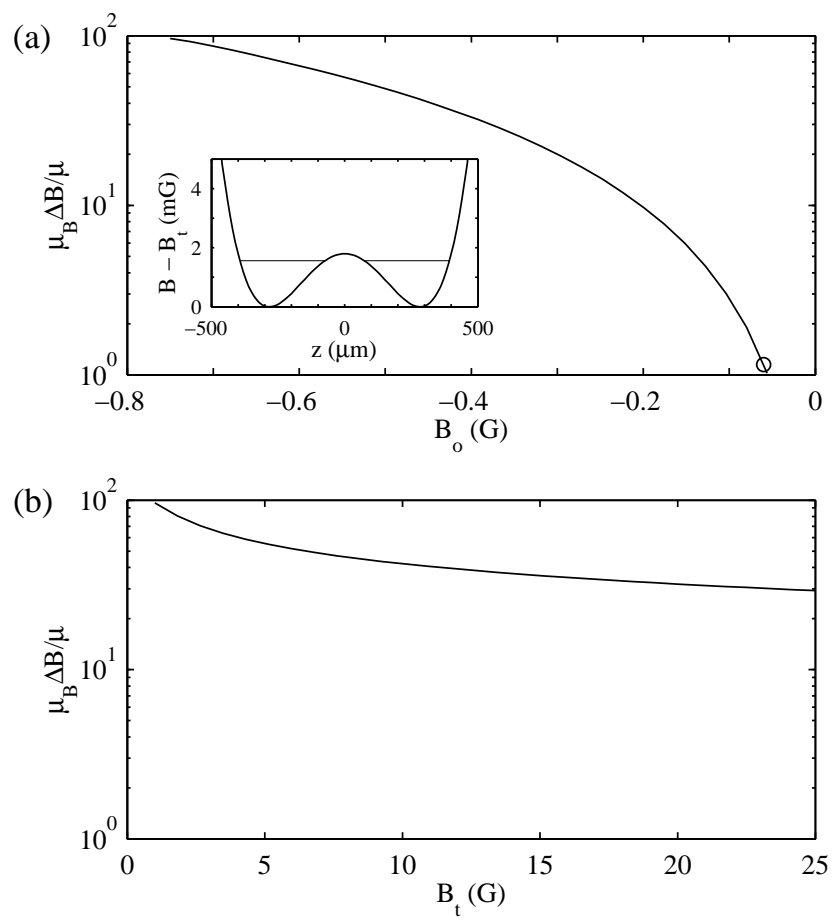

FIG. 6: (a) Control of the barrier height by altering the static bias field $B_{o}$ with $B_{t}=1 \mathrm{G}$. In the range shown, the ratio of barrier height and chemical potential drops from 100 to 1 . The circle indicates the parameters used for the inset. Inset: the magnetic potential for $B_{o}=-60 \mathrm{mG}$ and the chemical potential (horizontal lines) for $10^{6} \mathrm{Rb}$ atoms in each well. The calculation of $\mu$ is approximated by neglecting any effect of the condensate in the other well. (b) Control of the barrier height by altering the rotating bias field $B_{t}$ with $B_{o}=-0.75 \mathrm{G}$. Note that even at $25 \mathrm{G}$ the barrier height is still considerably greater than the chemical potential.

estimated by a numerical method where we integrate over the magnetic potential in the Thomas-Fermi approximation [44]. We keep the number of atoms constant at $10^{6}$ for each value of $B_{o}$ and solve for the chemical potential. Note that achieving very low barriers with this method requires very small values of $B_{o}$, which is discussed below.

We could also lower the barrier height by increasing $B_{t}$, as shown in Fig. 6(b). For our choice of parameters, using this method to make the barrier height equivalent to the chemical potential would involve increasing $B_{t}$ to approximately $1 \times 10^{5} \mathrm{G}$, and is therefore impractical. However, with a lower value of $B_{o}$, increasing $B_{t}$ offers greater sensitivity. For example, with $B_{o}=-110 \mathrm{mG}$ the barrier height and chemical potential become comparable when $B_{t}$ is approximately $20 \mathrm{G}$.

Achieving small well spacing also requires a very small bias field. For example, to obtain a well spacing of $2 z_{o}=300 \mu \mathrm{m}$ requires a static bias field of only
$-17 \mathrm{mG}$ for our choice of axial curvature. Note that $z_{o}=\sqrt{2\left|B_{o}\right| / B^{\prime \prime}}$, so that best control is achieved for largest possible curvatures and widely separated wells.

Achieving tight control of close well spacing and low barrier height therefore requires a high degree of coil current stability and shielding from external fields. High current power supplies of the sort often used for IP traps have relatively poor current stability, so that achieving close well separation with these is likely to be difficult. However, a low current example where a high degree of stability has been achieved is the QUIC trap [29]. The authors report in Ref. 445] reducing "residual fluctuations in the magnetic field to a level below $0.1 \mathrm{mG}$ ". Since the bias field required to achieve control over the well separation is small it should be possible to add a small external bias field without compromising the stability of a QUIC-style trap. It is worth noting that in the limit of a very small well spacing it becomes increasingly difficult to form a significant barrier, because this requires $B_{t} \ll B_{o}$. This condition leads to a very small $r_{o}$ and subsequent trap loss from Majorana spin-flips. Ensher has also found evidence [46] that an atomic cloud in a TOP trap with small $B_{t}$ is vulnerable to transitions to untrapped magnetic spin states, induced by residual AC magnetic fields associated with current noise.

\section{CONCLUSIONS}

We have developed a theoretical description for a double-well magnetic trap suitable for confining BoseEinstein condensates and cold thermal clouds. The separation of the wells is controlled by the static bias field $B_{o}$, while the barrier height also depends on the rotating bias field $B_{t}$. We have developed analytical forms of the potential that describe one or both wells, which are in good agreement with numerical simulations of the timeaveraged field. This trap has the convenient feature that it is based solely on magnetic fields and therefore avoids the issue of the relative stability of magnetic and optical fields addressed in [19]. It also offers a barrier height and well spacing that are tunable, so that a wide range of condensate phenomena may be accessible. Wide well separations are readily achieved and may be useful for interferometric applications, where a condensate in one well is perturbed and a second (local oscillator) is not. The double-TOP scheme also has the advantage that it is based on existing trapping technologies.

\section{Acknowledgments}

We are acknowledge the support of the Marsden Fund, contract UOO910, and the University of Otago. 
[1] M. H. Anderson et al., Science 269, 198 (1995).

[2] K. B. Davis et al., Phys. Rev. Lett. 75, 3969 (1995).

[3] C. C. Bradley, C. A. Sackett, J. J. Tollett, and R. G. Hulet, Phys. Rev. Lett. 75, 1687 (1995); 79, 1170 (1997).

[4] C. Menotti, J. R. Anglin, J. I. Cirac, and P. Zoller, Phys. Rev. A 63, 023601 (2001).

[5] R. W. Spekkens and J. E. Sipe, Phys. Rev. A 59, 3868 (1999).

[6] Y. Kagan, E. L. Surkov, and G. V. Shlyapnikov, Phys. Rev. A 54, R1753 (1996).

[7] J. Javanainen and M. Y. Ivanov, Phys. Rev. A 60, 2351 (1999).

[8] P. Capuzzi and E. S. Hernández, Phys. Rev. A 59, 3902 (1999).

[9] J. Javanainen, Phys. Rev. Lett. 57, 3164 (1986).

[10] F. Dalfovo, L. Pitaevskii, and S. Stringari, Phys. Rev. A 54, 4213 (1996).

[11] G. J. Milburn, J. Corney, E. M. Wright, and D. F. Walls, Phys. Rev. A 55, 4318 (1997).

[12] I. Zapata, F. Sols, and A. J. Leggett, Phys. Rev. A 57, R28 (1998).

[13] A. Smerzi, S. Fantoni, S. Giovanazzi, and S. R. Shenoy, Phys. Rev. Lett. 79, 4950 (1997).

[14] J. E. Williams, Phys. Rev. A 64, 013610 (2001).

[15] D. Jaksch et al., Phys. Rev. Lett. 86, 4733 (2001).

[16] K. Molmer, e-print cond-mat/0105533.

[17] L. Pitaevskii and S. Stringari, e-print cond-mat/0104458.

[18] J. F. Corney, G. J. Milburn, and W. Zhang, Phys. Rev. A 59, 4630 (1999).

[19] M. R. Andrews et al., Science 275, 637 (1997).

[20] S. Inouye et al., e-print cond-mat/0104444.

[21] D. E. Pritchard, Phys. Rev. Lett. 51, 1336 (1983).

[22] T. Bergeman, G. Erez, and H. J. Metcalf, Phys. Rev. A 35, 1535 (1987).

[23] V. Boyer et al., Phys. Rev. A 62, 021601(R) (2000).

[24] M.-O. Mewes et al., Phys. Rev. Lett. 77, 416 (1996).
[25] E. A. Burt et al., Phys. Rev. Lett. 79, 337 (1997).

[26] C. A. Sackett, C. C. Bradley, M. Welling, and R. G. Hulet, App. Phys. B 655, 433 (1997).

[27] L. V. Hau et al., Phys. Rev. A 58, R54 (1998).

[28] U. Ernst et al., Europhys. Lett. 41, 1 (1998).

[29] T. Esslinger, I. Bloch, and T. W. Hänsch, Phys. Rev. A 58, R2664 (1998).

[30] J. Söding et al., Appl. Phys. B 69, 257 (1999).

[31] D. G. Fried et al., Phys. Rev. Lett. 81, 3811 (1998).

[32] B. Desruelle et al., Phys. Rev. A 60, R1759 (1999).

[33] A. S. Arnold, C. MacCormick, and M. G. Boshier, e-print cond-mat/0103586.

[34] Y. Torii et al., Phys. Rev. A 61, 041602(R) (2000).

[35] C. Fort et al., Europhys. Lett. 49, 8 (2000).

[36] A. Robert et al., Science 292, 461 (2001).

[37] F. Pereira Dos Santos et al., Phys. Rev. Lett. 86, 3459 (2001).

[38] W. Ketterle, D. S. Durfee, and D. M. Stamper-Kurn, in Proceedings of the International School of Physics Enrico Fermi, edited by M. Inguscio, S. Stringari, and C. Wieman (IOS Press, Amsterdam, 1999), p. 67.

[39] A very similar double-well potential is used routinely when loading laser cooled atoms into a QUIC style IoffePritchard trap [29].

[40] W. Petrich, M. H. Anderson, J. R. Ensher, and E. A. Cornell, Phys. Rev. Lett. 74, 3352 (1995).

[41] K. B. Davis et al., Phys. Rev. Lett. 74, 5202 (1995).

[42] M. Kozuma et al., Phys. Rev. Lett. 82, 871 (1999).

[43] J. H. Müller et al., J. Phys. B 33, 4095 (2000).

[44] See, for example F. Dalfovo, S. Giorgini, L. P. Pitaevskii, and S. Stringari, Rev. Mod. Phys. 71, 463 (1999).

[45] I. Bloch, T. W. Hänsch, and T. Esslinger, Phys. Rev. Lett. 82, 3008 (1999).

[46] J. R. Ensher, Ph.D. thesis, University of Colorado (Boulder), 1998. 